\title{
Die Selbstdiffusionskoeffizienten des Antimons in Einkristallen
}

\author{
H. Cordes und K. KIM \\ Institut für Physikalische Chemie und Elektrochemie der Technischen Hochschule Braunschweig \\ (Z. Naturforschg. 20 a, 1197-1200 [1965]; eingegangen am 3. Juni 1965)
}

Mit einer Isotopenaustauschmethode wurden in Einkristallen die Selbstdiffusionskoeffizienten des Antimons parallel und senkrecht zur trigonalen Achse bestimmt. Die in einem Temperaturbereich von $500,0^{\circ} \mathrm{C}$ bis $630,2^{\circ} \mathrm{C}$ durchgeführten Messungen lassen sich folgendermaßen darstellen:

$$
\begin{aligned}
& D_{\perp \mathrm{c}}=(0,10 \pm 0,02) \exp \left[-(35,8 \pm 0,3) 10^{3} / R T\right] \mathrm{cm}^{2} \mathrm{sec}^{-1} \\
& D_{\pi \mathrm{c}}=(56 \pm 20) \exp \left[-(48,0 \pm 0,5) 10^{3} / R T\right] \mathrm{cm}^{2} \mathrm{sec}^{-1}
\end{aligned}
$$

Huntington, Ghate und Rosolowski ${ }^{1}$ geben in ihrer vor kurzem erschienenen Veröffentlichung ihre mit einer Tracermethode gemessenen Werte für die Selbstdiffusionskoeffizienten des anisotropen Antimons bekannt. Wir haben etwa gleichzeitig gleichartige Versuche durchgeführt, über deren Ergebnisse wir nachstehend berichten.

\section{Experimentelles}

Die Messungen wurden an Einkristallen vorgenommen, die unter Vorgabe eines zeitlich konstanten Temperaturgradienten mit definierter Absenkgeschwindigkeit aus extrem reinem Antimon hergestellt wurden. Die Ofentemperatur konnte bei der Einkristallherstellung mit Hilfe einer Ein-Aus-Regelung einer entsprechend dimensionierten Zusatzheizung während 24 Stunden selbst bei Temperaturen oberhalb der Schmelztemperatur des Antimons auf weniger als $0,1{ }^{\circ} \mathrm{C}$ konstant gehalten werden ${ }^{2}$, die Absenkgeschwindigkeit der weitgehend entgasten und vakuumgeschmolzenen Probe betrug $1 \mathrm{~cm} / \mathrm{Std}$., der Temperaturgradient in der Schmelzzone $15^{\circ} / \mathrm{cm}$. Bei Verwendung von Antimon mit 99,9999\% Feingehalt wurde - wie an anderer Stelle dargelegt wird $^{3}$ - eine Kristallgüte erhalten, die durch eine Versetzungsdichte längs des ganzen Kristallstabes in der (111)-Fläche von weniger als $10^{5} / \mathrm{cm}^{2}$ charakterisiert wird. Das allseitig zugeschmolzene, aus Supremaxglas gefertigte Schmelzgefäß wurde nach vorgenommener Kristallzüchtung mit Flußsäure ab-

1 H. B. Huntington, P. B. Ghate u. J. H. Rosolowski, J. Appl. Phys. 35, 3027 [1964].

2 R. Kruse, Dissertation, Technische Hochschule Braunschweig 1965.

3 H. Cordes u. R. Kruse, Z. Metallk., im Druck.

4 R. Madin u. W. R. Asher, Rev. Sci. Instr. 21, 881 [1950]. geätzt, um eine verformende mechanische Beanspruchung zu vermeiden. Das Schneiden der für die Messung der Diffusionskoeffizienten vorzubereitenden Proben erfolgte aus dem gleichen Grunde unter $\mathrm{Zu}$ hilfenahme einer Säuresäge ${ }^{4}$ mit einem FlußsäureSalpetersäure-Gemisch. Anschließend wurden die Proben elektrolytisch „wischpoliert" 5 . Die experimentell aufwendige Probenvorbereitung erscheint uns wesentlich, zumal MAdiN und $\operatorname{HiBaRD}^{6}$ zeigen konnten, daß bei selbst mit äußerster Sorgfalt vorgenommenem Zersägen und metallographischem und anschließendem chemischem Polieren der Einkristallprobe Verformungen des Kristalls zurückbleiben, selbst wenn die durch Polieren abgetragenen Oberflächenschichten relativ stark gewählt werden. $\mathrm{H}_{\mathrm{ART}}{ }^{7}$ hat darauf hingewiesen, daß die Versetzungen in Einkristallen die Volum-Selbstdiffusion merkbar beeinflussen können. TомızuкA ${ }^{8}$ hat die Wirkung von Versetzungen auf den Diffusionskoeffizienten experimentell bei der Diffusion von Antimon in Silbereinkristallen bei einer Temperatur von $600{ }^{\circ} \mathrm{C}$ untersucht und findet die von $\mathrm{H}_{\mathrm{ART}}{ }^{7}$ angegebene $\mathrm{Be}$ ziehung bestätigt. Williams und Slifkin ${ }^{9}$ deuten die bei der Diffusion von Gold in Silbereinkristallen beobachteten irregulären „Eindringungslinien“ als eine „Kurzschlußdiffusion“ entlang den Versetzungslinien. Die Diffusion entlang mikroskopischer Spaltrisse, die bei mechanischer Beanspruchung des spröden Antimonmetalls entstehen könnten, würde möglicherweise eine noch stärkere Kurzschlußdiffusion auslösen und dadurch die wahre Volum-Selbstdiffusion verfälschen.

5 G. Reinacher, Z. Methallk. 48, 162, 610 [1957].

6 R. Madin u. W. H. Hibard JR., Trans. AIME 185, 700 [1949].

7 E. W. Hart, Acta Met. 5, 597 [1957].

8 C. T. Tomizuka, Acta Met. 6, 660 [1958].

9 G. P. Williams u. L. Slifkin, Phys. Rev. Letters 1, 243 [1958]. 
Die mit der von uns vorgenommenen Bearbeitung für beide anisotropen Kristallagen erhaltenen Diffusionsproben - die trigonale Achse verläuft senkrecht zur (111)-Fläche der Antimonkristalle, die sich durch bevorzugte Spaltbarkeit längs dieser Fläche auszeichnet - wurden paarweise in einem Supremaxrohr mit einigen Milligramm radioaktivem Antimontrioxid zusammengebracht und nach längerem gründlichem Entgasen unter Vakuum abgeschmolzen (Restgasdruck $10^{-5}$ Torr). Nach beendetem Diffusionsglühen bei definierter Temperatur wurden die Proben nach Öffnen der Ampulle kurzzeitig in 49-proz. Flußsäure getaucht, um die an der Oberfläche adsorbierten Oxide abzulösen. Die parallel bzw. senkrecht zur trigonalen Achse orientierten Kristallproben wurden anschließend an beiden Stirnseiten mit einem dicht haftenden Chlorkautschuklack abgedeckt, während die übrigen Flächen der Proben zur Abtragung der durch Diffusion von radioaktivem Antimon von außen veränderten Oberflächenschichten in einer Polierlösung nach WernicK, Hobstetter, Lovell und Doris ${ }^{10}$ abgeätzt wurden. Nach mehrmaligem Wässern und Entfernung der Lackschicht wurden die getrockneten Proben bis auf ihre Stirnseiten lackgeschützt und die Radioaktivität jeweils der Stirnseiten mit einer Szintillationssonde und einem Einkanalimpulszähler Typ GS-DMZ der Firma Siemens bestimmt. Gemessen wird die $\gamma$-Strahlung des ${ }^{124} \mathrm{Sb}$ von $0,603 \mathrm{MeV}$. Der totale Absorptionskoeffizient des Antimons für diese Strahlung dürfte $0,58 \mathrm{~cm}^{-1}$ betragen. Nach Bestimmung der Gesamtaktivität $R_{\mathrm{T}}$ wurde eine dünne, etwa $10 \mu$ betragende Oberflächenschicht durch chemisches Polieren abgetragen und die Restaktivität ermittelt. Dieses Verfahren wurde fortgesetzt, solange die Restaktivität $R_{x}$ im Vergleich zu dem Nulleffekt noch genügend große Impulszahlen lieferte. Die jeweils durch Polieren abgetragenen Schichtdicken wurden aus den auf $\pm 10 \mu \mathrm{g}$ bestimmten Gewichtsabnahmen der Probe ermittelt. Der mittlere statistische Fehler der Restaktivitäten $R_{x}$ beträgt durchweg etwa $1 \%$. Die Werte für die Diffusionskoeffizienten $D$ wurden mit Hilfe der Beziehung ${ }^{11}$

$$
R_{x 1}: R_{x 2}=F_{x 1}: F_{x 2}
$$

mit $\quad F_{x}=\frac{2}{\sqrt{\pi}} \exp \left(-\frac{x^{2}}{4 D t}\right)-\frac{x}{\sqrt{D t}} \operatorname{erfc} \frac{x}{2 \sqrt{D t}}$

10 J. H. Wernick, J. N. Hobstetter, L. C. Lovell u. D. Doris, J. Appl. Phys. 29, 1013 [1958].

11 J. J. Rohan, N. E. Pickering u. J. Kennedy, J. Electrochem. Soc. 106, 705 [1959]. berechnet, wobei die $F_{x}$-Werte einem Tabellenwerk entnommen wurden ${ }^{12}$. Die mittlere Streuung der $D$-Werte bei 8 Versuchen ist im Durchschnitt 6,7\% bei $D_{\perp \text { c }}$ bzw. $8,5 \%$ bei $D_{\| \mathrm{c}}$. Der Diffusionsverlauf ist in Abb. 1 und 2 dargestellt, wobei $N_{\mathrm{T}}$ bzw. $N_{x}$ die von $R_{\mathrm{T}}$ und $R_{x}$ umgerechneten diffundierten $\mathrm{Ge}-$ samt- bzw. Restatomzahlen bei $x$ bedeuten. Es besteht eine recht gute Übereinstimmung der experimentellen mit dem aus den gemittelten $D$-Werten zu erwartenden theoretischen Diffusionsverlauf ${ }^{11}$.

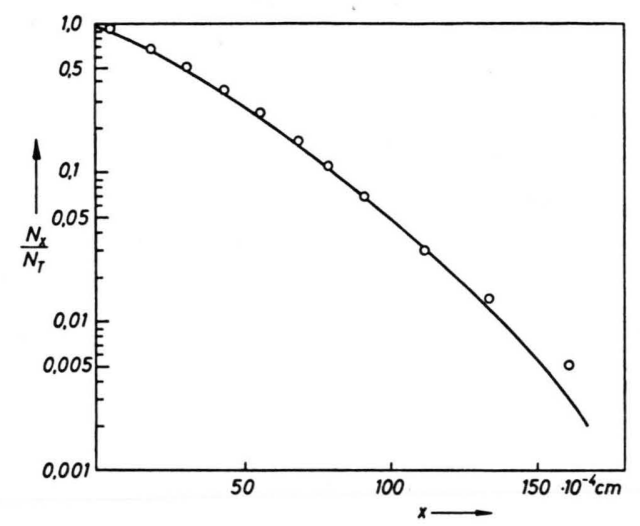

Abb. 1. Diffusionsverlauf für $D_{\perp \text { c }}$ bei einer Diffusionsdauer von $24 \mathrm{Std}$. bei $627,7^{\circ} \mathrm{C}$. 000 experimentell, — theoretisch.

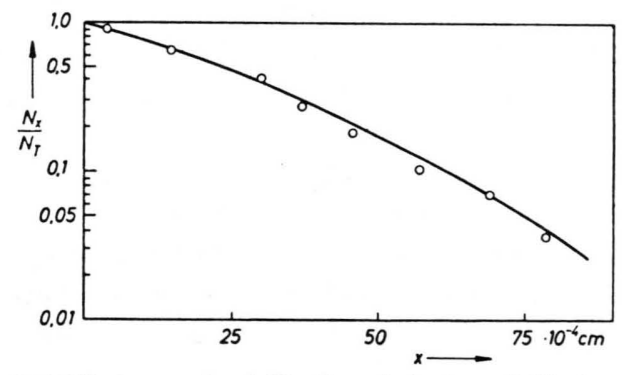

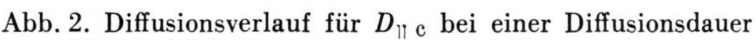
von 24 Std. bei $627,7^{\circ} \mathrm{C}$. 000 experimentell, $\longrightarrow$ theoretisch.

\section{Ergebnisse und Diskussion}

Die gemessenen Werte der Selbstdiffusionskoeffizienten senkrecht und parallel zur trigonalen Achse $D_{\perp \text { c }}$ und $D_{\| \text {c }}$ sind in Tab. 1 und in Abb. 3 aufgetragen. Es besteht eine die Beziehung

$$
D=D_{0} \exp (-Q / R T)
$$

befriedigende Abhängigkeit. Die Werte für $Q$ bzw. $D_{0}$ und deren mittlere Streuung wurden mit Hilfe

12 H. S. Carlslaw u. J. C. JäGer, Conduction of Heat, At the Clarendon Press, Oxford 1959. 


\begin{tabular}{|c|c|c|}
\hline $\begin{array}{c}T \\
{ }^{\circ} \mathrm{C}\end{array}$ & $\begin{array}{c}D_{\perp \mathrm{c}} \\
\mathrm{cm}^{2} / \mathrm{sec}\end{array}$ & $\begin{array}{c}D_{\text {॥c }} \\
\mathrm{cm}^{2} / \mathrm{sec}\end{array}$ \\
\hline 630,2 & $(2,28 \pm 0,13) \cdot 10^{-10}$ & $(1,42 \pm 0,13) \cdot 10^{-10}$ \\
& & $(1,30 \pm 0,15) \cdot 10^{-10}$ \\
627,7 & $(2,13 \pm 0,18) \cdot 10^{-10}$ & $(1,18 \pm 0,14) \cdot 10^{-10}$ \\
& $(2,30 \pm 0,18) \cdot 10^{-10}$ & $(1,24 \pm 0,11) \cdot 10^{-10}$ \\
620,0 & $(1,74 \pm 0,06) \cdot 10^{-10}$ & $(1,05 \pm 0,13) \cdot 10^{-10}$ \\
& $(1,88 \pm 0,17) \cdot 10^{-10}$ & $(1,01 \pm 0,10) \cdot 10^{-10}$ \\
590,0 & $(8,84 \pm 0,51) \cdot 10^{-11}$ & $(3,59 \pm 0,38) \cdot 10^{-11}$ \\
& $(9,17 \pm 0,45) \cdot 10^{-11}$ & \\
550,0 & $(3,30 \pm 0,16) \cdot 10^{-11}$ & $(1,06 \pm 0,08) \cdot 10^{-11}$ \\
& $(3,22 \pm 0,20) \cdot 10^{-11}$ & $(8,73 \pm 0,51) \cdot 10^{-12}$ \\
540,0 & $(2,35 \pm 0,13) \cdot 10^{-11}$ & $(7,19 \pm 0,11) \cdot 10^{-12}$ \\
& $(2,27 \pm 0,27) \cdot 10^{-11}$ & \\
510,0 & $(1,03 \pm 0,09) \cdot 10^{-11}$ & $(2,01 \pm 0,07) \cdot 10^{-12}$ \\
& $(1,16 \pm 0,03) \cdot 10^{-11}$ & $(2,19 \pm 0,06) \cdot 10^{-12}$ \\
500,0 & $(8,28 \pm 0,34) \cdot 10^{-12}$ & 1,64 \\
& $(7,94 \pm 0,74) \cdot 10^{-12}$ & \\
\hline
\end{tabular}

Tab. 1. Zusammenstellung der Ergebnisse.

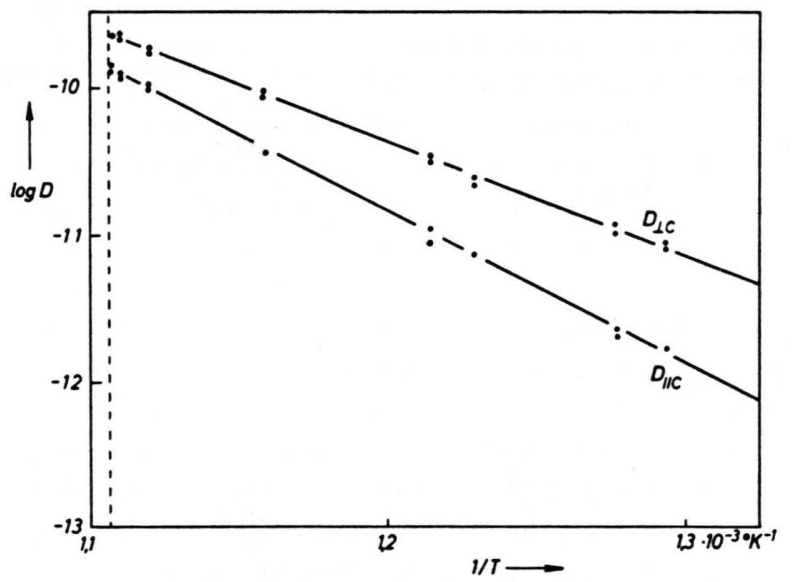

Abb. 3. Temperaturabhängigkeit der Selbstdiffusion.

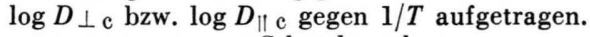
- - Schmelzpunkt.

der Ausgleichsrechnung bestimmt. Im Temperaturbereich von $500{ }^{\circ} \mathrm{C}$ bis $0,3{ }^{\circ} \mathrm{C}$ unterhalb des Schmelzpunktes gilt

$D_{\perp \mathrm{c}}=(0,10 \pm 0,02) \exp \left[-(35,8 \pm 0,3) \cdot 10^{3} / R T\right] \mathrm{cm}^{2} \mathrm{sec}^{-1}$, $D_{\| \mathrm{c}}=(56 \pm 20) \exp \left[-(48,0 \pm 0,5) \cdot 10^{3} / R T\right] \mathrm{cm}^{2} \mathrm{sec}^{-1}$.

Die Anisotropie des Selbstdiffusionskoeffizienten von Antimon ist relativ gering und nimmt mit zunehmender Temperatur ab. Die beiden $D$-Werte unterscheiden sich jedoch selbst $0,3{ }^{\circ} \mathrm{C}$ unterhalb des Schmelzpunktes noch deutlich um einen außer-

13 S. Umino, Sci. Rep. Tohoku Imp. Univ. 15, 604 [1904].

14 F. Wüst, A. Meuten u. R. Dürrer, Forschungsarb. Gebiet Ingenieurwes. 204, 40 [1928].

15 W. Klemm u. H. Niermann, Angew. Chem. 75, 508 [1963].

16 C. Zener, J. Appl. Phys. 22, 372 [1951] ; Acta Cryst. 3, 346 [1950]. halb der Fehlergrenzen der Messungen liegenden Faktor von der Größenordnung 5/3.

Antimon ist wie Arsen und Wismut durch eine besonders hohe Schmelzentropie ausgezeichnet. Dieser Umstand muß mit einer beim Schmelzen dieser Elemente erfolgenden sehr weitgehenden Umorientierung der Atome in beiden Phasen in Verbindung gebracht werden. Die experimentelle Bestimmung der Schmelzentropie ist - soweit die Messung kalorimetrisch durchgeführt wird - eine Differenzmessung. Der Temperaturverlauf der Enthalpie des Festkörpers und der Schmelze wird beidseitig des Schmelzpunktes linear bis zur Phasenumwandlungstemperatur extrapoliert und die Enthalpiedifferenz der Schmelzenthalpie gleichgesetzt. Eine genauere Bestimmung des Temperaturkoeffizienten der Enthalpie von festem und geschmolzenem Antimon von Umino $^{13}$, von Wüst, Meuten und Dürrer ${ }^{14}$ und von Klemm und NiermanN ${ }^{15}$ läßt jedoch erkennen, daß eine lineare Extrapolation in der Nähe des Schmelzpunktes nicht gerechtfertigt ist. Sowohl kurz unterhalb und ausgeprägter oberhalb der als Schmelzpunkt bezeichneten Temperatur steigt die Enthalpie stärker als linear an. Der Temperaturverlauf der Enthalpie der unterkühlten Schmelze von Antimon läßt darauf schließen, daß unterhalb des Schmelzpunktes exotherme Prozesse ablaufen, die im Sinne einer Art „nachträglicher Kristallisation" als eine nachgeholte Ausbildung des stabilen Schichtgitters gedeutet werden könnten. Es erscheint deshalb nicht ausgeschlossen, daß andererseits oberhalb des Schmelzpunktes die uneingeschränkt isotrope Atomverteilung nicht unmittelbar eintritt, sondern daß über den Schmelzpunkt hinaus die Schmelze Teilbereiche eines anisotropen Gitterkomplexes enthält. $\mathrm{Zu}$ ähnlichen Folgerungen haben Dichte- und Enthalpiebestimmungen bei Gallium geführt, das im festen Zustand ebenso durch ein anisotropes Gitter gekennzeichnet ist.

Die Frage nach der Art des Diffusionsmechanismus bedarf u. E. eingehenderer weiterer Untersuchungen. Auf Grund der verschiedentlich ergänzten und verbesserten Theorie von $Z_{\mathrm{ENER}}{ }^{16}$ besteht allgemein die Auffassung, daß mit wenigen Ausnahmen, wie z. B. Chrom ${ }^{17}$ und $\gamma$-Uran ${ }^{18}$, für die meisten kubisch

17 H. W. Paxton u. E. G. Gondolf, Arch. Eisenhüttenwes. 30, 55 [1959].

18 S. H. Rothman, L.T. Lloyd u. A. L. Harkness, J. Metals 12, 68 [1960]. 
kristallisierenden Metalle eine Diffusion über einen Leerstellenmechanismus vorliegen dürfte. Als Begründung hierfür wird durchweg der Wert des vorexponentiellen Faktors bei der üblichen exponentiellen Darstellung angeführt, der mit rechnerisch ermittelten Größen verglichen wird, die in Anwendung der Theorie des Übergangszustandes auf Grund eines für den Kristalltyp jeweils vereinfachten Modells erhalten wurden. Als Parameter enthalten diese Modellrechnungen empirisch gewonnene Materialdaten (meist die elastischen Konstanten), und Kristalldefekte (wie Versetzungen, Korngrenzen, Poren, Spaltrisse) und allgemein innere Oberflächen bleiben unberücksichtigt. Dabei kann es keinem Zweifel unterliegen, daß gerade solche Kristalldefekte einen weitgehenden Einfluß auf die experimentellen Meßwerte, insbesondere des Diffusionskoeffizienten, haben dürften.

Pawel und Lundy ${ }^{19}$ konnten bei der Messung der Diffusion von Niob in Tantaleinkristallen zeigen, daß die Oberflächendiffusion längs einzelner Kanäle die Meßwerte, die für die Randzonen der Kristalle erhalten wurden, stark verändert. Andelin, Knight und $\mathrm{K}_{\mathrm{AHN}}{ }^{20}$ geben eine Zusammenstellung der experimentell bestimmten Werte des Selbstdiffusionskoeffizienten von Wolfram, die von verschiedenen Bearbeitern mit teilweise unterschiedlichen Meßverfahren erhalten wurden. Dabei läßt sich die Unsicherheit erkennen, mit der aus den Zahlenwerten der Versuchsdaten gemittelte Werte sowohl des vorexponentiellen Faktors als auch der "Aktivierungsenthalpie" ermittelt werden können. Bei den einzelnen Angaben differiert z.B. der vorexponentielle Faktor zwischen 0,54 und $6,3 \cdot 10^{7}$ ! Analoge Zusammenstellungen an anderen Beispielen bestätigen die Streuung der entsprechenden Werte. Dieser Umstand ist u.E. nicht zuletzt darauf zurückzuführen, daß den Messungen im allgemeinen wegen der vielfältigen und zumeist unkontrollierbaren Defektstellen im Matrixkristall eine notwendig beträchtliche Streubreite zukommt und die "Aktivierungsentropie“ und "-Enthalpie“ nicht eindeutige Parameter des Elementarschrittes des jeweiligen Diffusionsmechanismus, als vielmehr Mittelwerte sind, die sich aus der Überlagerung unterschiedlicher und anteilig verschiedener Mechanismen ergeben.

Wir möchten glauben, daß bei der zumeist nur

19 R. E. Pawel u. T. S. Lundy, Acta Met. 13, 345 [1965].

20 R. L. Andelin, J. D. Knight u. M. Kahn, Trans. Met. Soc. AIME 233, 19 [1965]. geringen theoretischen Differenzierung zwischen den einzelnen Fehlstellen- und Ringmechanismen für die Volumdiffusion die Experimente an weitgehend versetzungsfrei gezogenen Einkristallen im Einzelfall erst eine zweifelsfreiere Entscheidung bezüglich des Mechanismus zulassen.

Durch Vergleich mit hinreichend gesichert erscheinenden Ergebnissen an anderen Metallen besteht u. E. nach einiger Grund zu der Annahme, daß die Diffusion in der zur trigonalen Achse senkrechten Ebene im Antimon über einen Leerstellenmechanismus verläuft, wohingegen Hinweise für den Elementarschritt in anderen Kristallrichtungen einstweilen fehlen. Wir erwarten, daß eine z. Zt. vorgenommene Ergänzung der vorliegenden Untersuchung unter Einschluß von Messungen der Druckabhängigkeit der Selbstdiffusionskoeffizienten an Antimon und Wismut zusätzliche Kriterien zur Entscheidung des Diffusionsmechanismus erbringt.

Der Vergleich der Ergebnisse der vorliegenden Arbeit mit denen einer Untersuchung von Huntington, Ghate und Rosolowski ${ }^{1}$ über die gleichfalls an Ein. kristallen gemessenen Selbstdiffusionskoeffizienten des anisotropen Antimons bestärkt uns in der Vorsicht, den Zahlenwerten des vorexponentiellen Faktors und der "Aktivierungsenthalpie“ ohne weiteres eine unmittelbare Beziehung zu den Elementarschritten des Diffusionsvorgangs beizumessen. Huntington und Mitarbeiter weisen darauf hin, daß die Sprödigkeit des Antimons und insbesondere die bevorzugte Spaltbarkeit in Richtung senkrecht zur trigonalen Achse, die auf die Schichtstruktur des Kristalls zurückgeht, die beträchtliche Fehlerbreite der Messungen bedingt. Tab. 2 enthält die aus den Meß-

\begin{tabular}{|c|c|c|c|c|}
\hline \multirow{2}{*}{} & \multicolumn{2}{|c|}{ HuntingtoN } & \multicolumn{2}{c|}{ diese Arbeit } \\
\cline { 2 - 5 } & $\ln D_{0}$ & $\begin{array}{r}Q(\mathrm{kcal} / \\
\mathrm{g} \text { Atom })\end{array}$ & $\ln D_{0}$ & $\begin{array}{r}Q(\mathrm{kcal} / \\
\mathrm{g} \text { Atom })\end{array}$ \\
\hline$D_{\text {॥c }}$ & $3,1 \pm 0,7$ & $\mathbf{4 7 , 1} \pm 1,2$ & $4,0 \pm 0,3$ & $48,0 \pm 0,5$ \\
$D_{\perp \mathrm{c}}$ & $2,8 \pm 2,8$ & $\mathbf{4 4 . 4 \pm 4 , 9}$ & $-2,3 \pm 0,2$ & $35,8 \pm 0,3$ \\
\hline
\end{tabular}

Tab. 2. Meßdaten der Selbstdiffusion nach Huntington ${ }^{1}$ und der vorliegenden Arbeit.

daten von Huntington und Mitarbeitern und von uns gemittelten Größen $\ln D_{0}$ und $Q$ der exponentiellen Darstellung der Temperaturfunktion der Selbstdiffusionskoeffizienten von Antimon.

Die Untersuchung wurde durch Mittel des Herrn Bundesministers für wissenschaftliche Forschung ermöglicht, wofür auch an dieser Stelle gedankt sei. 